\title{
Will peptides make vaccines?
}

\section{The prospect of basing a new generation of vaccines on synthetic peptides keeps cropping up. Despite the promise, there is a long way to go.}

SINCE proteins are relatively rigid structures, or so the crystallographers would have us believe, it would be unreasonable to expect that short runs of amino acids exposed on the surface of proteins would be held in the same conformation as that preferred by the same peptide when free in solution. By the same token, it would be unreasonable to expect that antibodies raised against a free peptide would frequently recognize the protein of which the peptide was part. Reason, however, has been confounded by facts. The frequency with which antibodies against short peptides, less than twenty amino acids long, react against the parent proteins is turning out to be remarkably high (see, for example, Niman et al., Proc. natn. Acad. Sci. U.S.A. 80, 4949; 1983). And so effective are some of the peptides as antigens that much effort is going into the design and synthesis of peptides that will serve as vaccines, stimulating the production of antibodies against the proteins on the surface of pathogenic microorganisms.

The attractions of peptide vaccines are clear. They would do away with the need to compose vaccines from inactivated preparations or avirulent strains of pathogenic microorganisms. They would avoid the necessity of a source of biological material, the blood of infected patients for example, from which antigenic proteins are prepared as the basis of a subunit vaccine. And they would allow the biotechnological process of using genetically-manipulated bacteria or yeasts to produce the antigenic proteins of vaccines to be replaced by a process of organic synthesis of peptides. So much for the theory.

In practice, there are many problems to be solved before any specific peptide vaccine is developed, the first of which is to identify an appropriate peptide. Generally that is a case of analysing the amino acid sequence (nowadays usually deduced from a DNA sequence) of the protein in question by a combination of the methods available for predicting which segments would be on the surface of the folded molecule. One favoured combination is first to identify helical stretches and then to find which of those contain well separated hydrophobic and hydrophilic sides, an indication that one side is on the surface face of the protein. By such means, highly antigenic peptides that induce protective antibodies against the foot and mouth disease virus have been identified and are in the process of being evaluated as vaccines.

A similar approach to the production of peptide vaccines against poliovirus was very recently reported by Emini et al. (Nature 304, 699; 1983), whose work is complementary to a more biological approach already noted in these columns $(304,395 ; 1983)$. The biological approach, pursued in Britain at the National Institute for Biological Standards and Control in collaboration with the University of Leicester, is to generate mutants of the poliovirus that are no longer neutralized by an antibody that is effective against the original virus, and then to determine which changes in amino acid sequence on the surface protein of the mutants are responsible for their escape from the antibody. That has led to the identification of a target on the poliovirus VP1 protein for one neutralizing antibody (a neutralization epitope of the protein in immunological jargon). The target is confined to an eight amino acid peptide that is therefore a strong candidate as a peptide vaccine.

That peptide (as part of an eleven amino acid peptide) is one of five synthesized by Emini et al. and tested for their potential as vaccines. Four of the five were recognized by a poliovirus neutralizing antibody but the only one that was able to induce neutralizing antibodies when injected into rabbits was the peptide that incorporates the eight amino acid stretch incriminated biologically. That, therefore, is the only one of the five whose further potential as a peptide vaccine is worth exploring. All is not lost for the potential use of the other five peptides, say Emini et al. , because each of them, although incapable of inducing neutralizing antibodies in rabbits, does stimulate the immune system of the animals in such a way as to potentiate their antibody response to a subsequent injection of poliovirus. That kind of priming of the immune system by peptides may be an alternative or an adjunct to vaccination with the pathogen.

For poliovirus, as for foot and mouth disease virus, effective vaccines already exist and any peptide vaccine will have to be measured against them both in protective and commercial terms. The position is very different for the Plasmodium parasites that cause malaria and against which there are no vaccines, although a start has been made this year by cloning the genes for the surface proteins of two different stages of Plasmodium. On page 29 of this issue,
Godson et al. take matters a stage further and, along the way, demonstrate that malaria is an excellent candidate for a peptide vaccine. Their efforts have been directed towards the cell surface of the sporozoite stage of the malaria parasite, the stage in which the parasite passes from mosquito to vertebrate (monkey in this case). DNA sequencing of that part of the gene for the major sporozoite protein that encodes its antigenic determinant has revealed a remarkable structure, in which a 36 base pair sequence is almost exactly repeated twelve times in tandem. There is only one variation in bases that would affect the twelve-amino acid peptide encoded by the repeated sequences.

Chemical synthesis of the peptides that correspond to either one or two identical repeats was followed by very preliminary tests of their potential as a peptide vaccine. Both were shown to be recognized by monoclonal antibodies directed against the sporozoite surface antigen.

So far, so good, but there is a very long way to go to show that an effective vaccine can really be developed from the peptide (not least because the project has to be translated from monkey to man, probably an easy task, and because it has yet to be demonstrated that the sporozoite stage of the parasite is an appropriate target, which is dubious).

It is, in every case, going to be a long slog from the identification of a candidate peptide to the production of an effective vaccine and many of the projects will fall by the wayside. There will need to be a great deal of testing of different peptides presented in different ways (for example, bound to carriers, polymerized or cyclized) to maximize the immune response. It is unlikely that any peptide vaccine will be useful in the prevention of human diseases unless it is given in conjunction with an adjuvant to boost the immune response, and since there are no acceptable adjuvants, much effort will need to be expended on their development. Finally, there will be such scientifically mundane questions as shelf life and temperature stability. Most of the progress in all these areas will not make exciting reading and is unlikely to appear in Nature. Indeed, much of it will be safely in the hands of pharmaceutical companies and unlikely to appear anywhere. What we can look forward to from them is news of a successful peptide vaccine, with foot and mouth disease the most likely to benefit first.

Peter Newmark 